\title{
Simulation of two-phase nanofluid flow and heat transfer in a 3D diamond shape cavity equipped with square shaped obstacle and decreasing dimensions
}

\author{
Amin Goldanlou ${ }^{1}$, Alireza Aghaei ${ }^{2}$, Amin Dezfulizadeh ${ }^{3}$, sara rostami ${ }^{4}$, and Suvanjan \\ Bhattacharyya $^{5}$ \\ ${ }^{1}$ Duy Tan University \\ ${ }^{2}$ University of Kashan \\ ${ }^{3}$ Islamic Azad University of Arak \\ ${ }^{4}$ Ton Duc Thang University \\ ${ }^{5}$ Birla Institute of Technology and Science
}

May 26, 2020

\begin{abstract}
The present study investigates numerically symmetry simulation of two-phase nanofluid flow and heat transfer in a 3D diamond shape cavity equipped with square-shaped obstacle and decreasing dimensions. The studied material in the present study is assumed with two different emissivity values, $=0.3$ and $=0.9$, due to analyze the effects of emissivity values on radiation heat transfer. Also two different Rayleigh numbers, $\mathrm{Ra}=106$ and 108. The heat transfer fluid is water-based Cu nanofluid which makes a Newtonian nanofluid, but other base fluid is also analyzed. The main aim of present work is to simulate the 3D diamond shape cavity equipped with square-shaped obstacle and decreasing dimensions geometry using symmetry method and also investigate the effects of different Rayleigh numbers, emissivity values and different nanoparticles volume concentrations on thermal and hydraulic characteristics of the model. Base on obtained results, by an increase of nanoparticles volume concentration the temperature gradients and heat transfer characteristics are improved but the streamlines have not a significant change and by an increase of nanoparticles volume concentration the temperature gradients and heat transfer characteristics are improved, but the streamlines have not a significant change. According to results at higher Rayleigh numbers, the heat transfer characteristics are enhanced. Also, it is found that higher Rayleigh numbers and nanoparticles volume concentrations lead to more heat transfer inside the cavity and changes in emissivity coefficients have not a significant effect on heat transfer characteristics and nanofluid flow in the cavity.
\end{abstract}

\section{Hosted file}

Goldanlou et al-Cavity Nanofluid-rev.docx available at https://authorea.com/users/325502/ articles/454369-simulation-of-two-phase-nanofluid-flow-and-heat-transfer-in-a-3ddiamond-shape-cavity-equipped-with-square-shaped-obstacle-and-decreasing-dimensions 


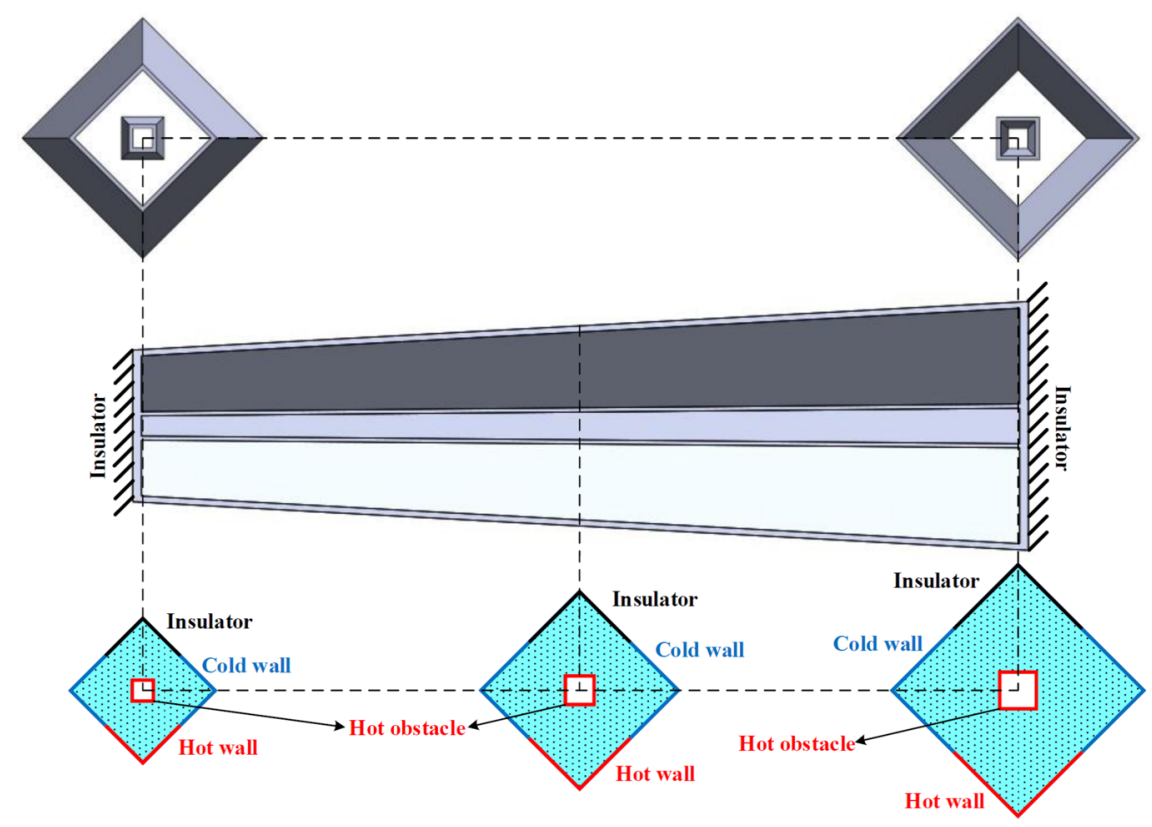

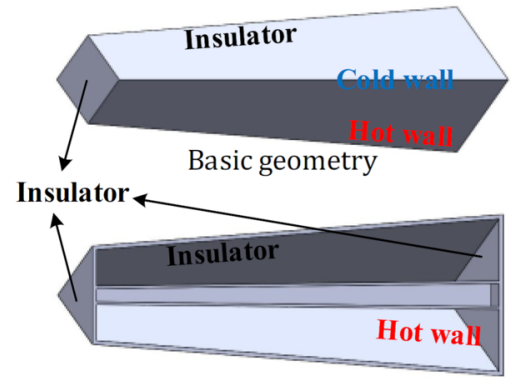

Simulated geometry in present study using Symmetry boundary condition

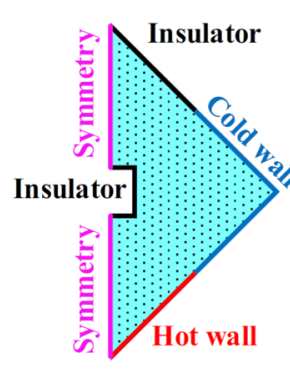

(a)

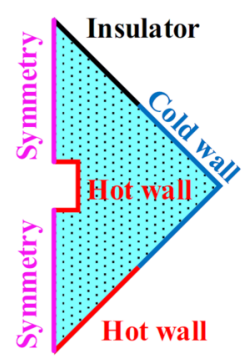

(b) 

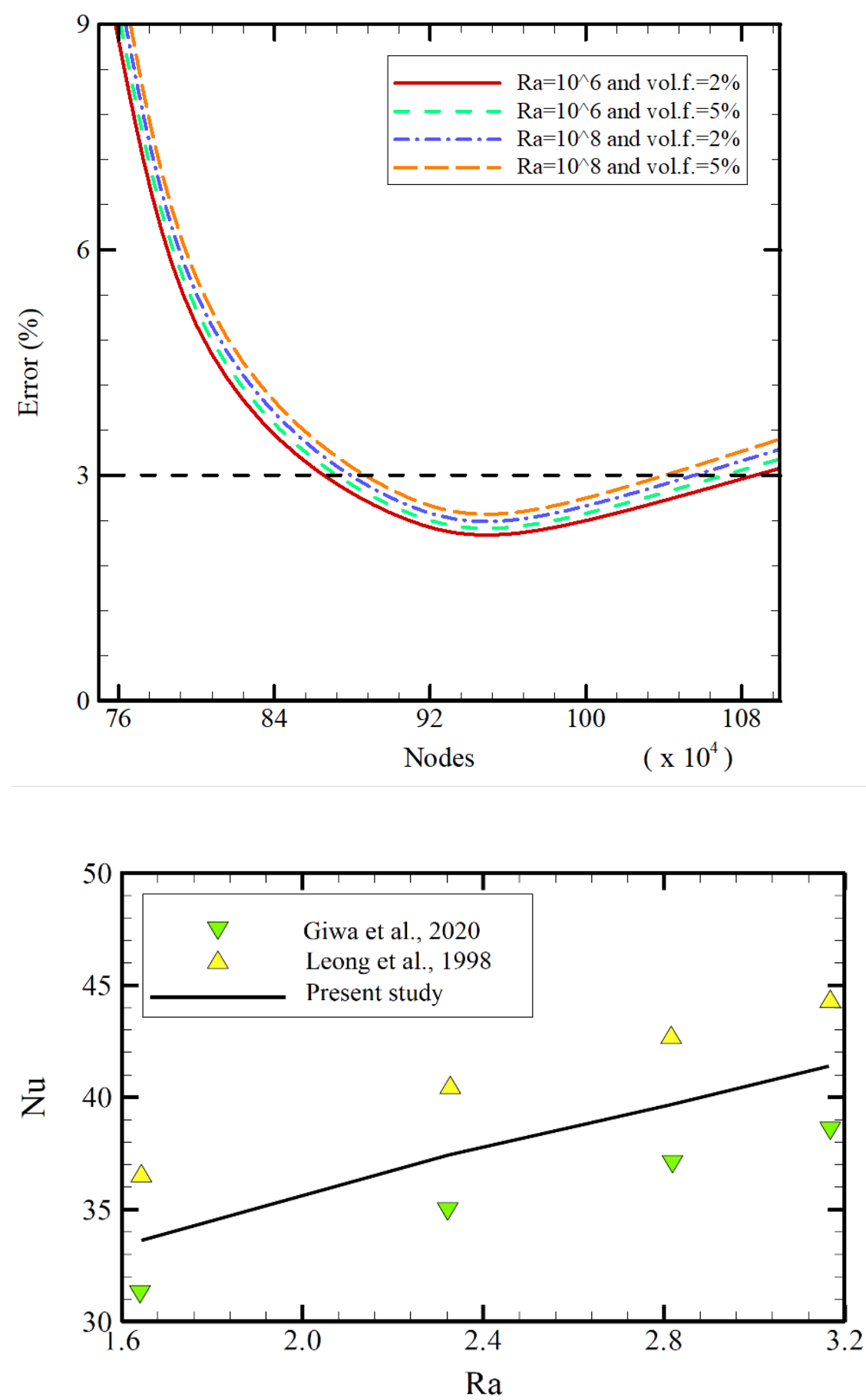

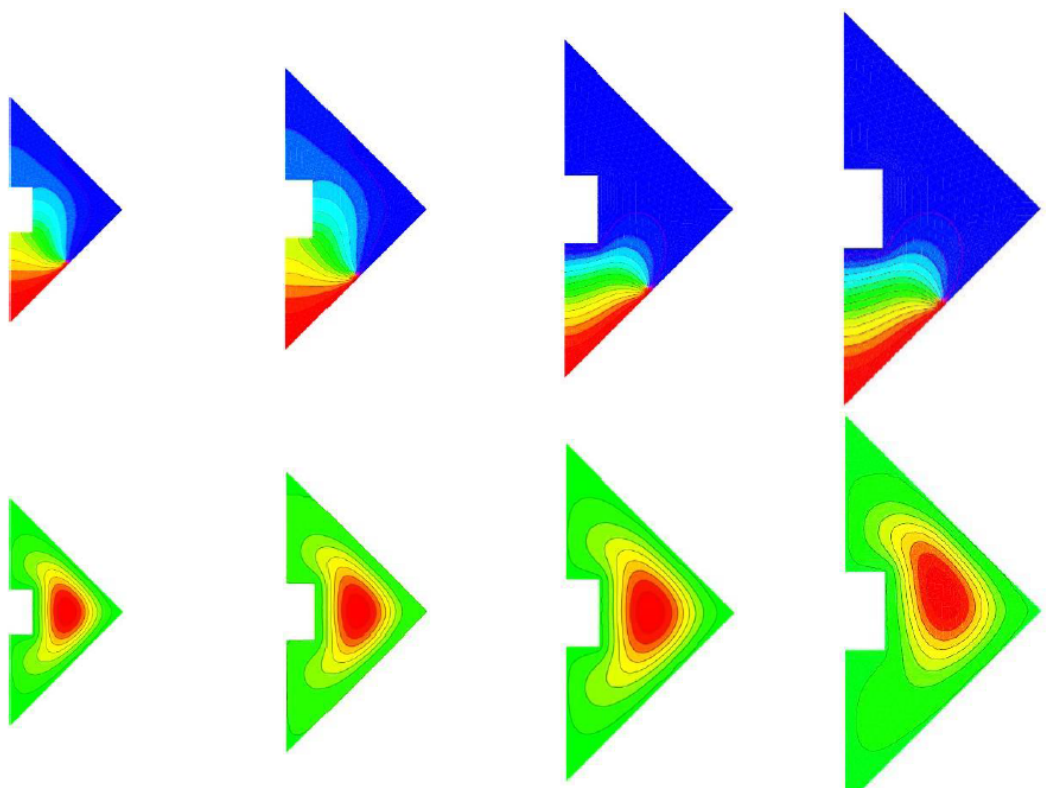

$x=10 \mathrm{~mm}$

$x=340 \mathrm{~mm}$

$x=680 \mathrm{~mm}$

$\mathrm{Ra}=10^{6} \& \varepsilon=0.3 \& \phi=1 \%$

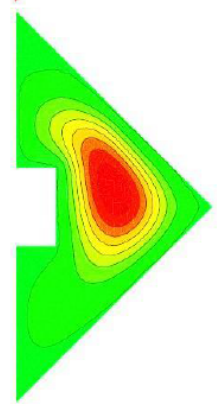

$x=990 \mathrm{~mm}$
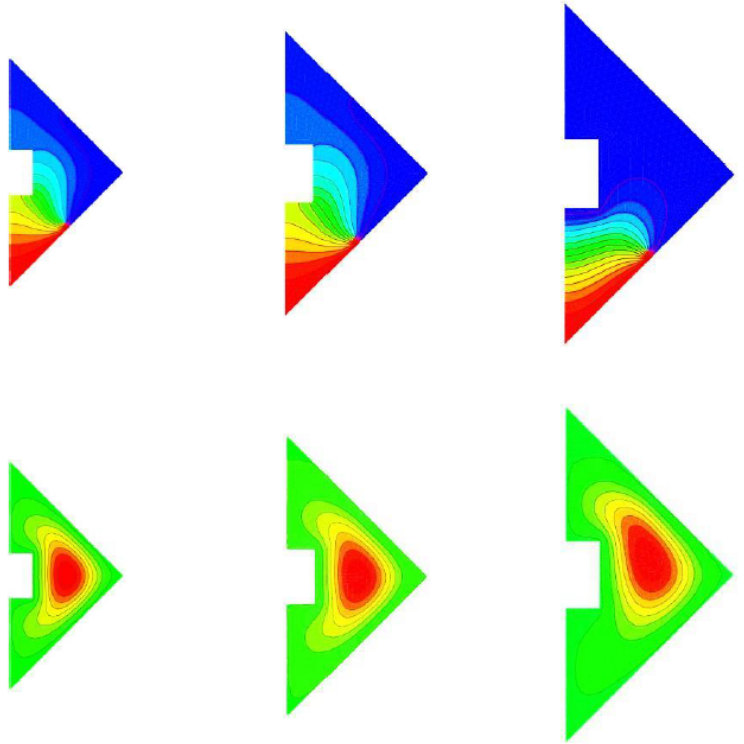

$x=10 \mathrm{~mm}$

$\begin{array}{cc}x=340 \mathrm{~mm} & x=680 \mathrm{~mm} \\ \mathbf{R a}=\mathbf{1 0} \mathbf{0}^{6} \boldsymbol{\&} \boldsymbol{\varepsilon}=\mathbf{0 . 3} \boldsymbol{\&} \boldsymbol{\phi}=\mathbf{5 \%}\end{array}$

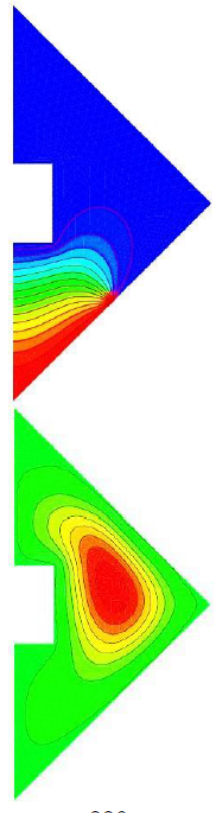

$x=990 \mathrm{~mm}$ 

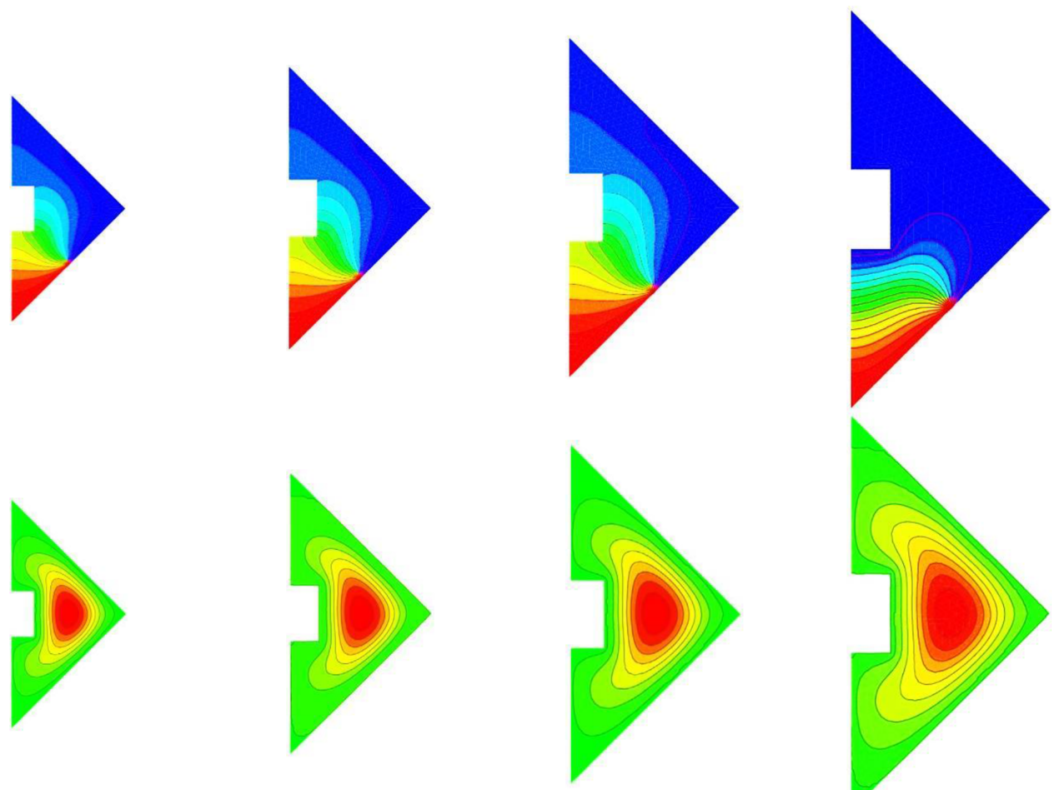

$x=10 \mathrm{~mm}$

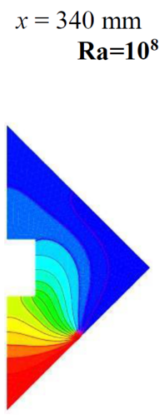

$x=680 \mathrm{~mm}$

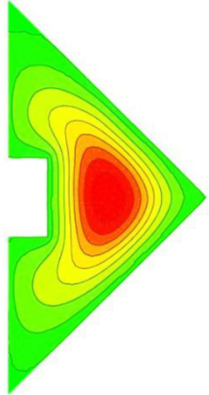

$x=990 \mathrm{~mm}$
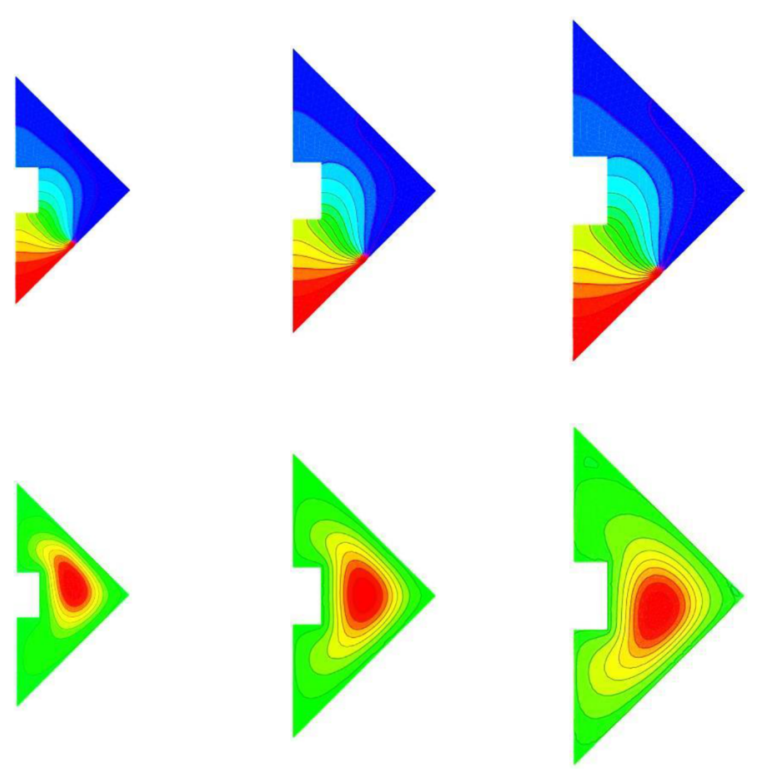

$x=10 \mathrm{~mm}$
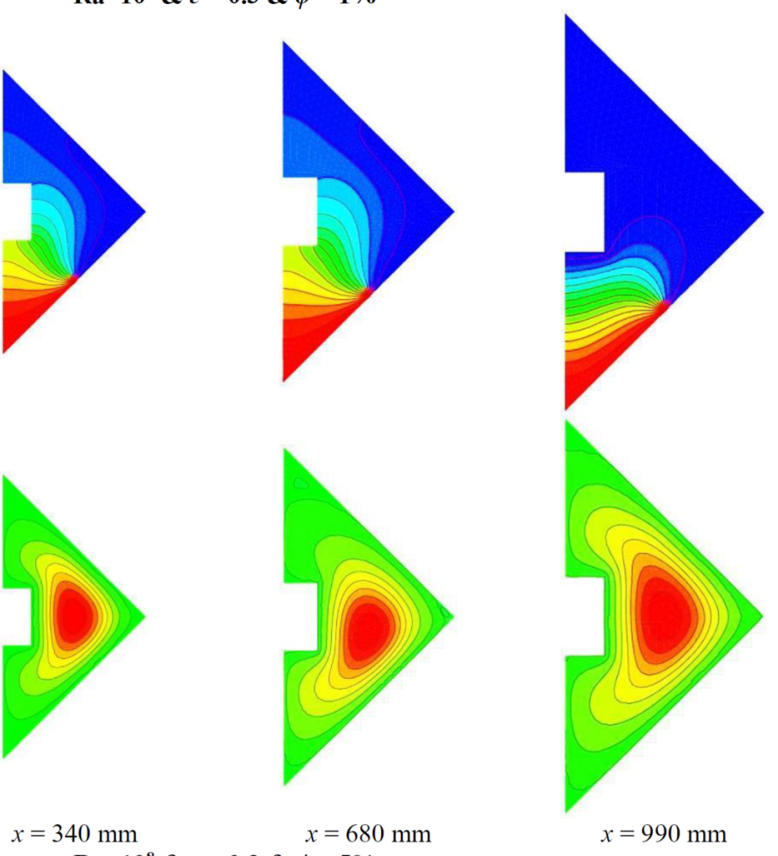

$x=990 \mathrm{~mm}$

$\mathrm{Ra}=10^{8} \& \varepsilon=0.3 \& \phi=5 \%$ 

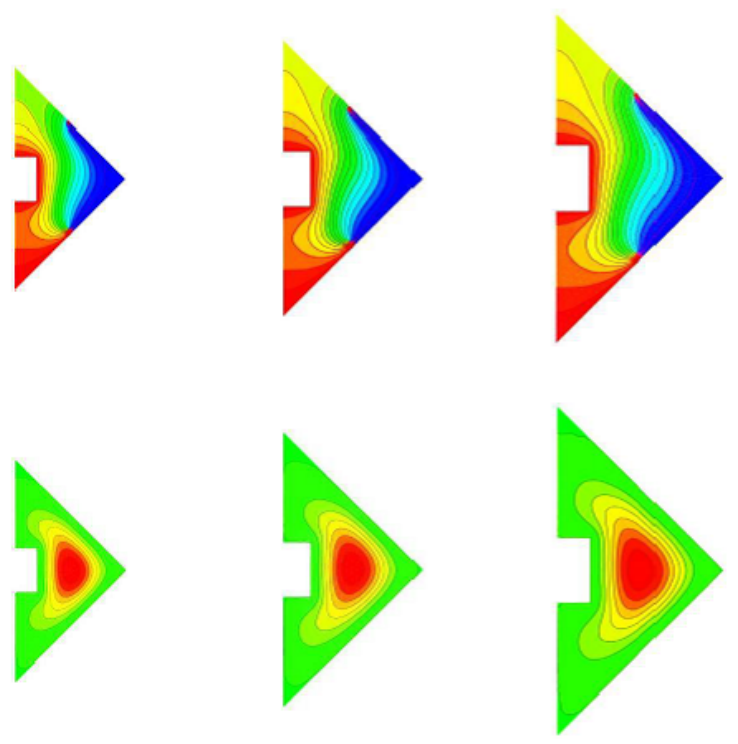

$x=10 \mathrm{~mm}$

$x=340 \mathrm{~mm}$ $\mathrm{Ra}=10^{6} \& \varepsilon=0.3 \& \quad x=680 \mathrm{~m}$
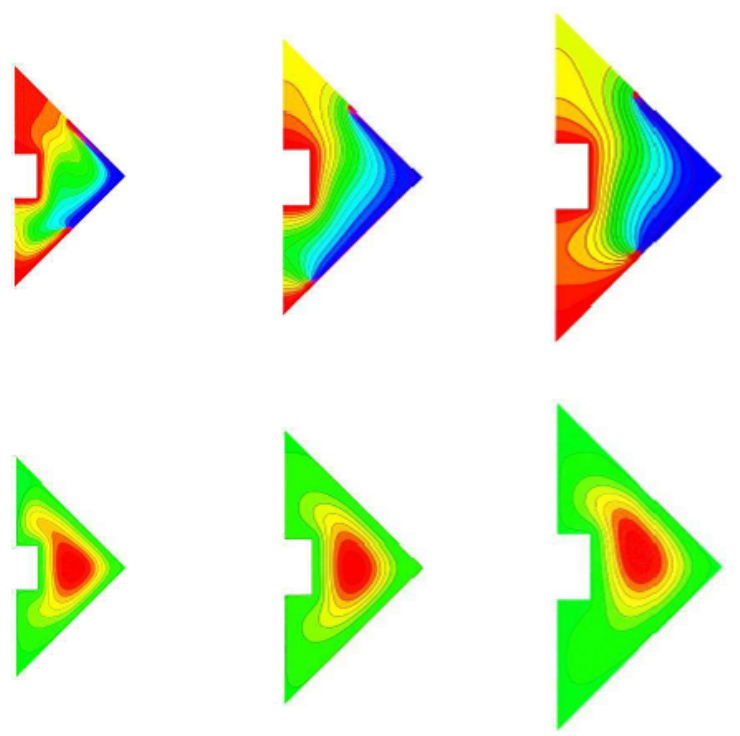

$x=10 \mathrm{~mm}$

$x=340 \mathrm{~mm}$ Ra $=10^{6} \& \varepsilon=0.3 \& \quad \begin{array}{r}x=680 \mathrm{~m} \\ \phi=5 \%\end{array}$

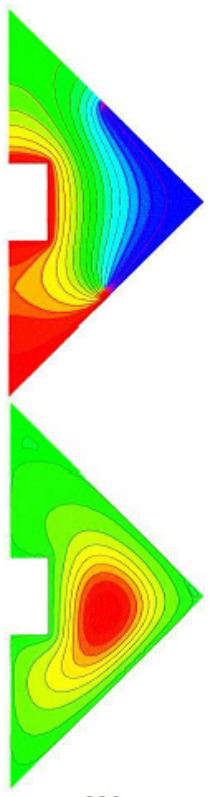

$x=990 \mathrm{~mm}$

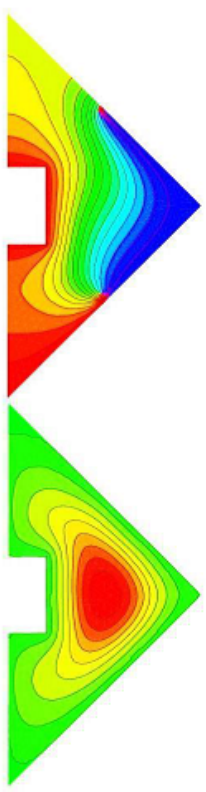

$x=990 \mathrm{~mm}$ 

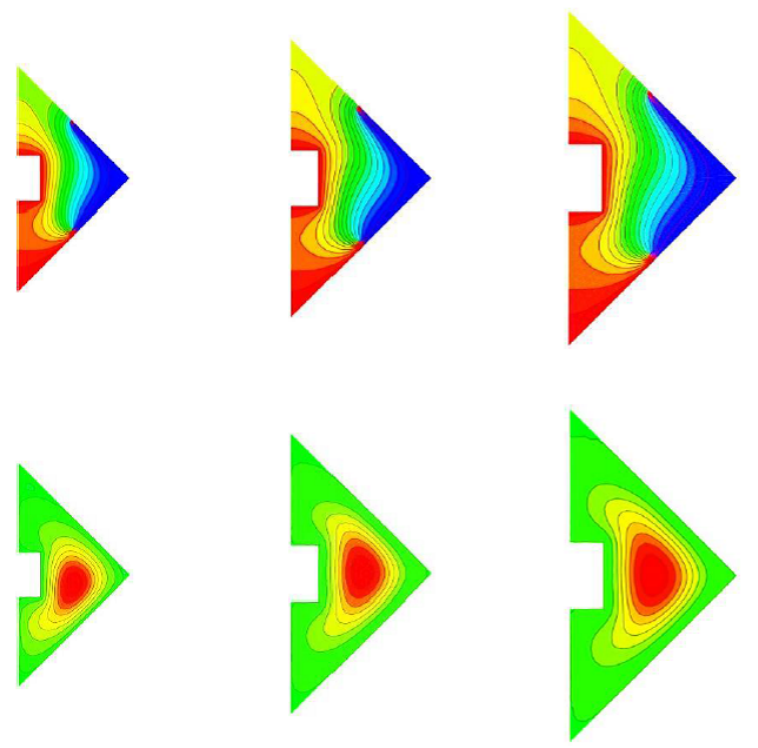

$x=10 \mathrm{~mm}$

$$
x=340 \mathrm{~mm}
$$

$x=680 \mathrm{~mm}$

$\mathrm{Ra}=10^{8} \& \varepsilon=0.3 \& \phi=1 \%$
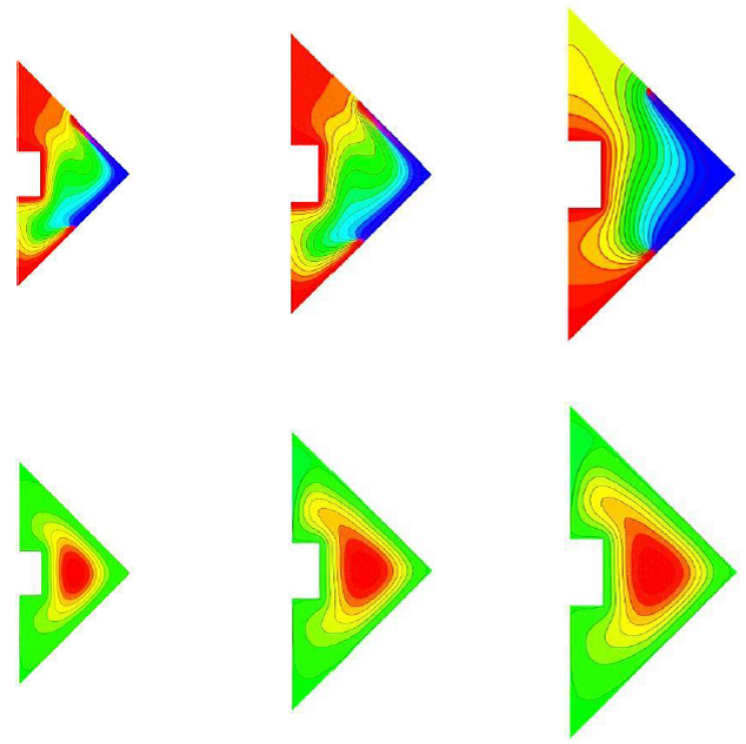

$x=10 \mathrm{~mm}$

$x=340 \mathrm{~mm} \quad x=680 \mathrm{~mm}$

$\mathrm{Ra}=10^{8} \& \varepsilon=0.3 \& \phi=5 \%$

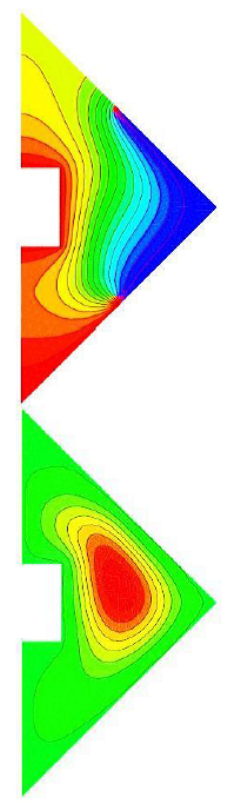

$x=990 \mathrm{~mm}$
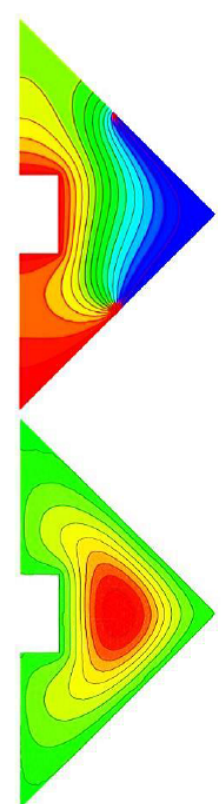

$x=990 \mathrm{~mm}$ 

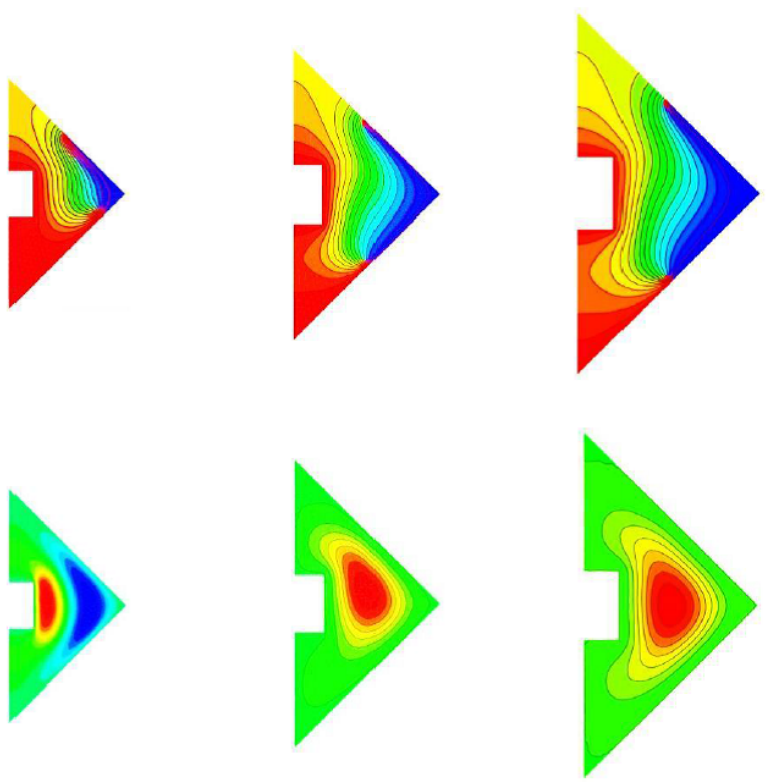

$x=10 \mathrm{~mm}$

$x=340 \mathrm{~mm}$

$x=680 \mathrm{~mm}$

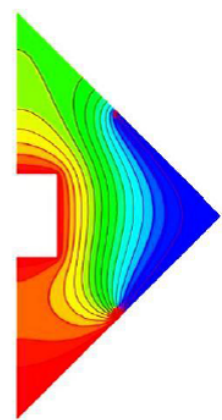

$\mathrm{Ra}=10^{6} \& \varepsilon=0.3 \& \phi=1 \%$
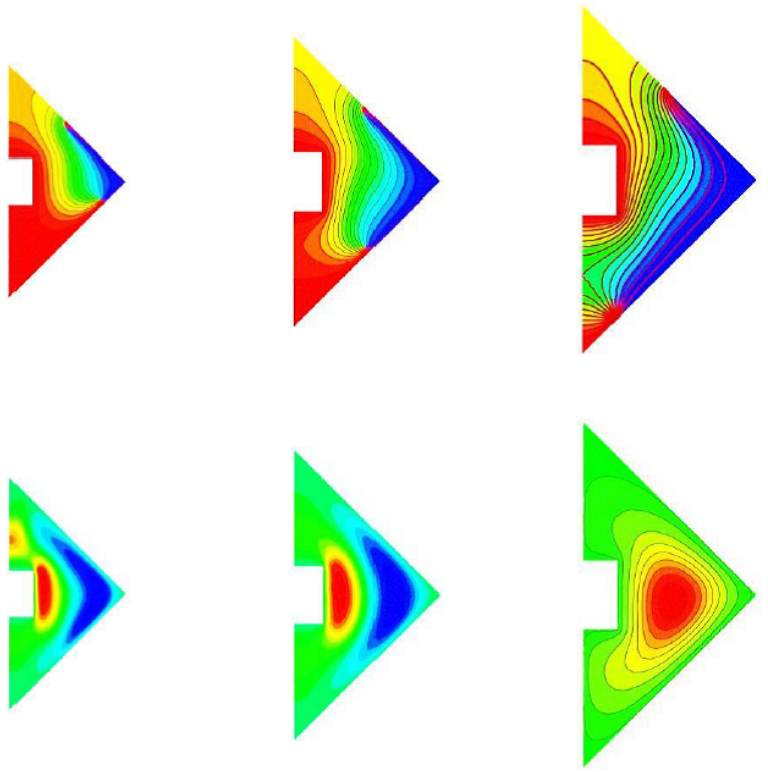

$x=10 \mathrm{~mm}$

$x=340 \mathrm{~mm}$

$x=680 \mathrm{~mm}$

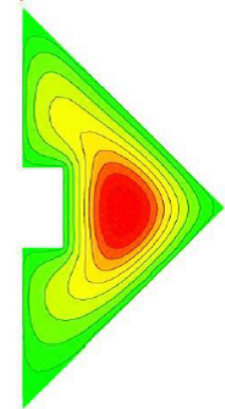

$x=990 \mathrm{~mm}$
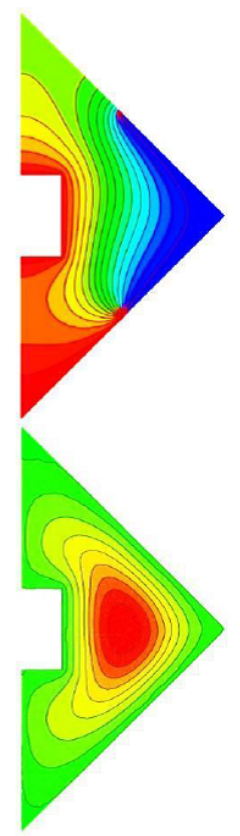

$x=990 \mathrm{~mm}$

$\mathrm{Ra}=10^{6} \& \varepsilon=0.3 \& \phi=5 \%$ 

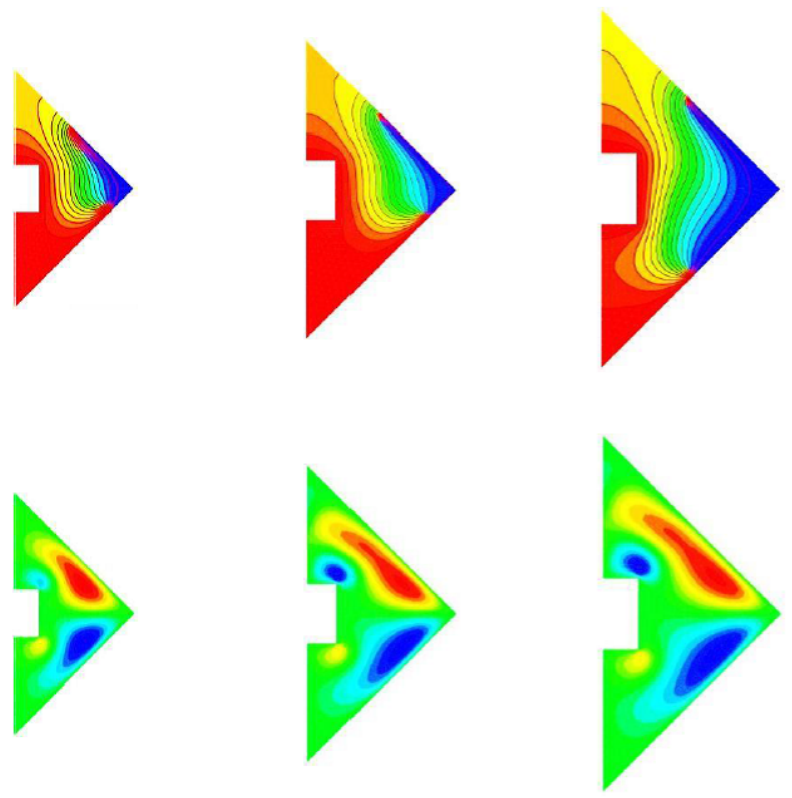

$x=10 \mathrm{~mm}$

$x=340 \mathrm{~mm}$

$x=680 \mathrm{~mm}$

$\mathrm{Ra}=10^{8} \& \varepsilon=0.3 \& \phi=1 \%$
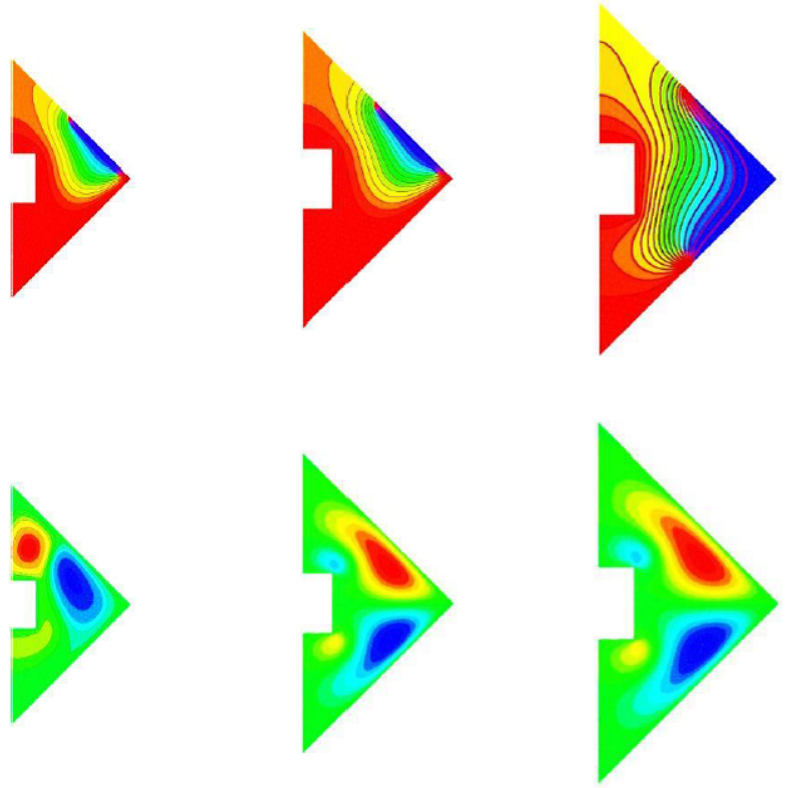

$x=10 \mathrm{~mm}$

$x=340 \mathrm{~mm}$

$x=680 \mathrm{~mm}$
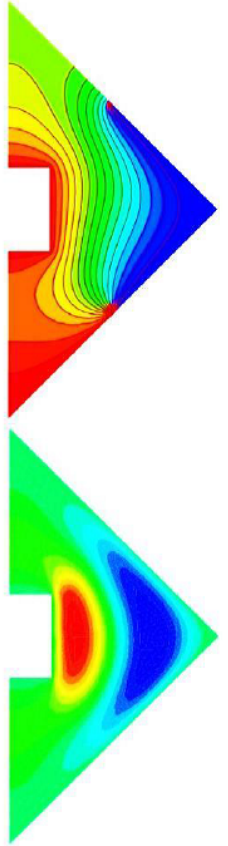

$x=990 \mathrm{~mm}$

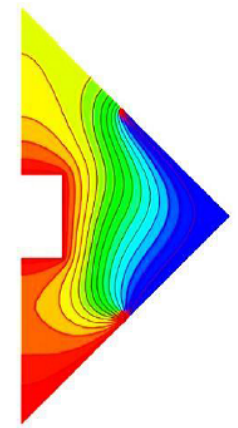

$\mathrm{Ra}=10^{8} \& \varepsilon=0.3 \& \phi=5 \%$ 

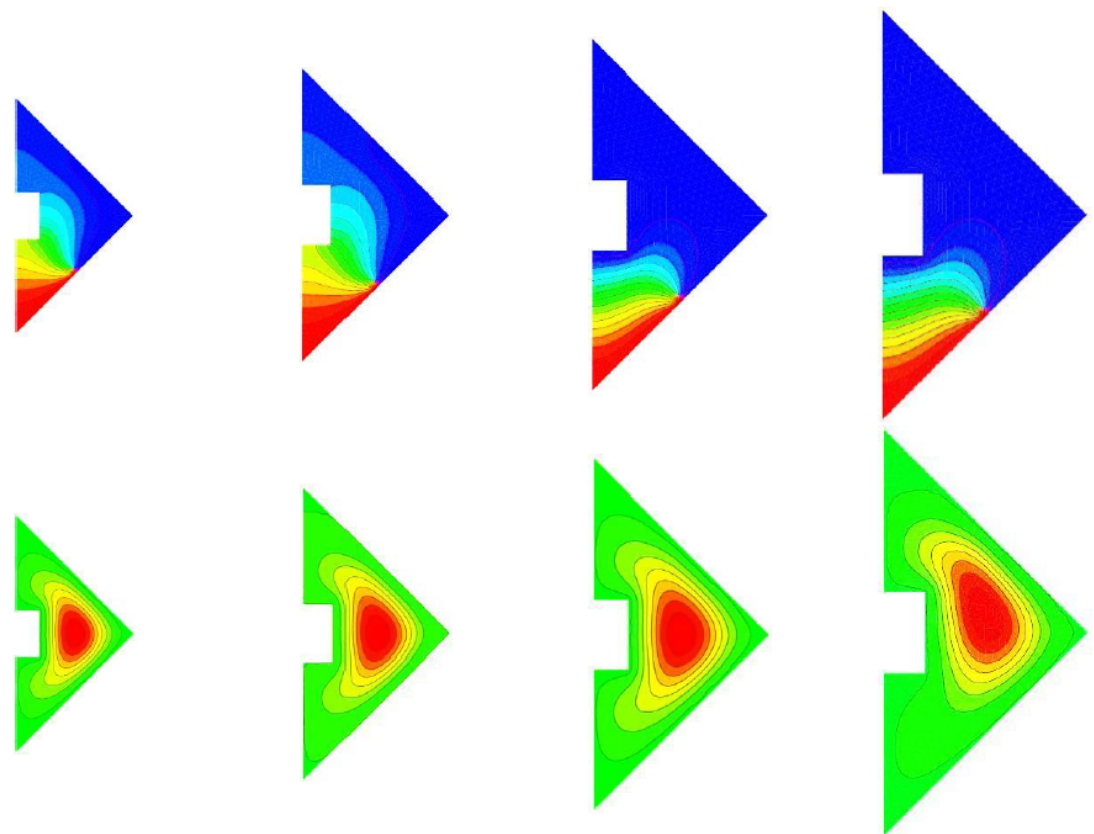

$x=10 \mathrm{~mm}$

$x=340 \mathrm{~mm}$

$x=680 \mathrm{~mm}$

$x=990 \mathrm{~mm}$

$\mathrm{Ra}=10^{6} \& \varepsilon=0.9 \& \phi=1 \%$
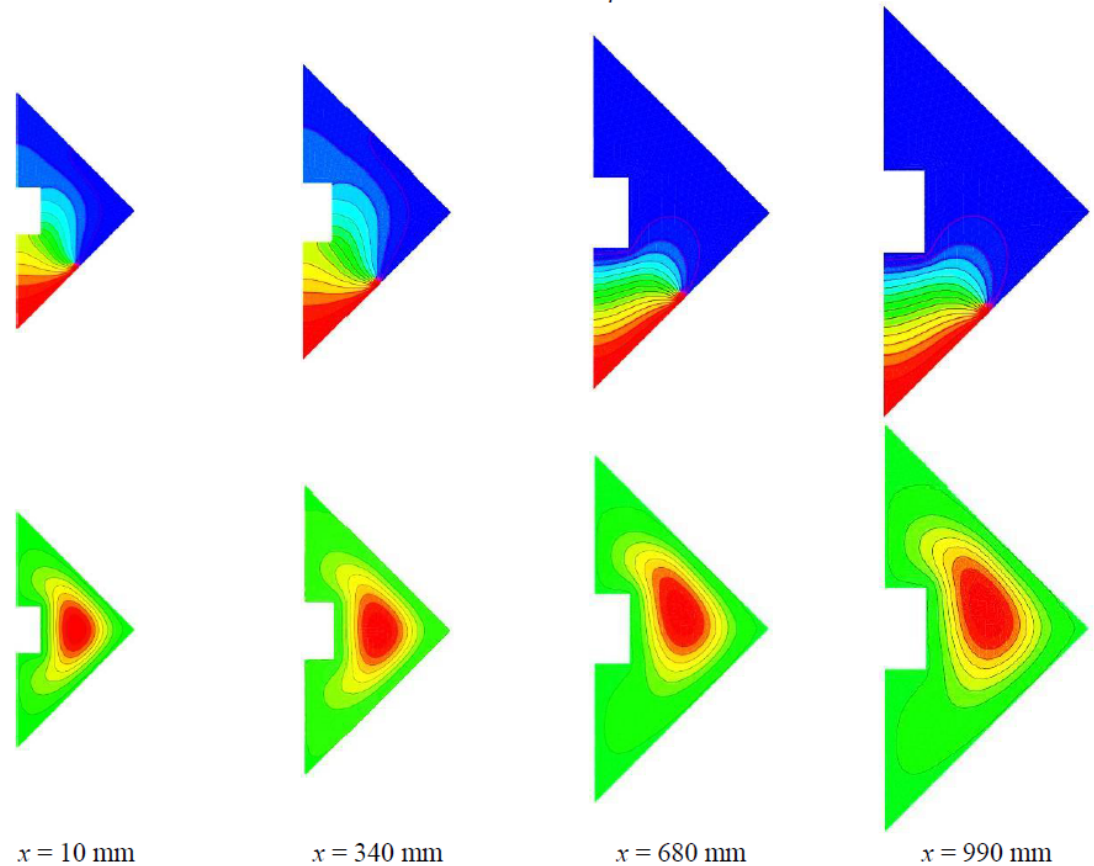

$x=10 \mathrm{~mm}$

$x=340 \mathrm{~mm}$

$x=680 \mathrm{~mm}$

$x=990 \mathrm{~mm}$

$\mathrm{Ra}=10^{6} \& \varepsilon=0.9 \& \phi=5 \%$ 

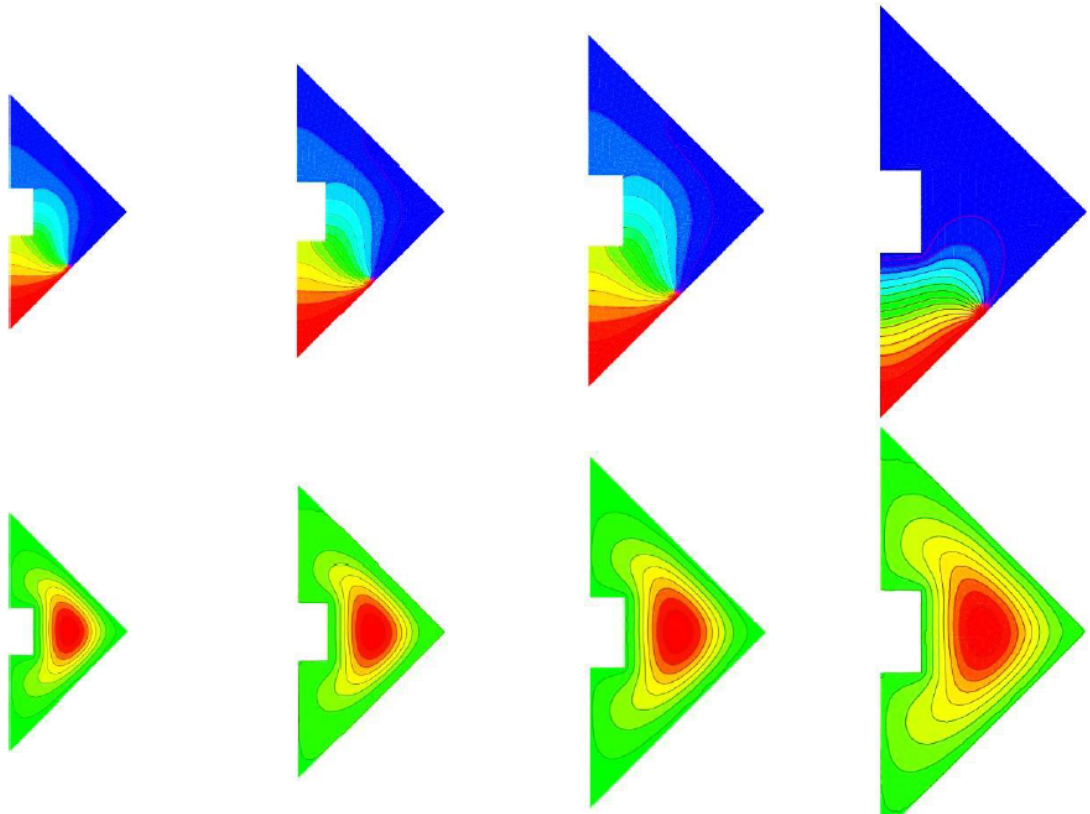

$x=10 \mathrm{~mm}$

$x=340 \mathrm{~mm}$

$x=680 \mathrm{~mm}$

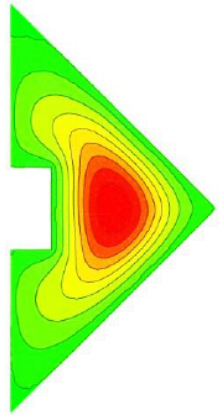

$\mathrm{Ra}=10^{8} \& \varepsilon=0.9 \& \phi=1 \%$
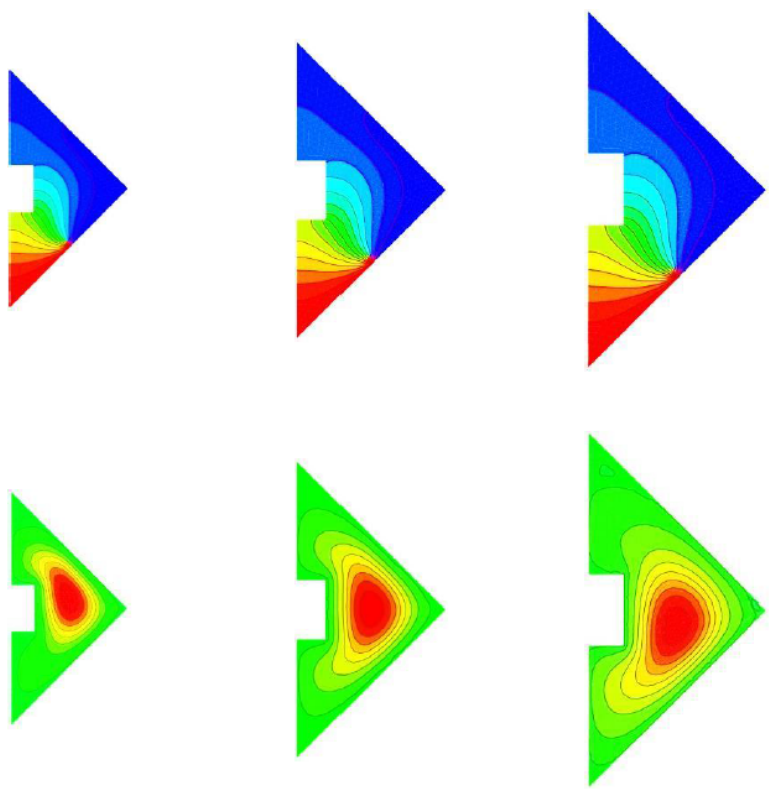

$x=10 \mathrm{~mm}$

$x=340 \mathrm{~mm}$

$x=680 \mathrm{~mm}$
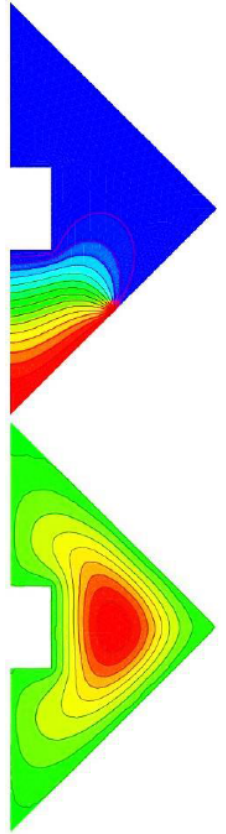

$x=990 \mathrm{~mm}$

$\mathrm{Ra}=10^{8} \& \varepsilon=0.9 \& \phi=5 \%$ 

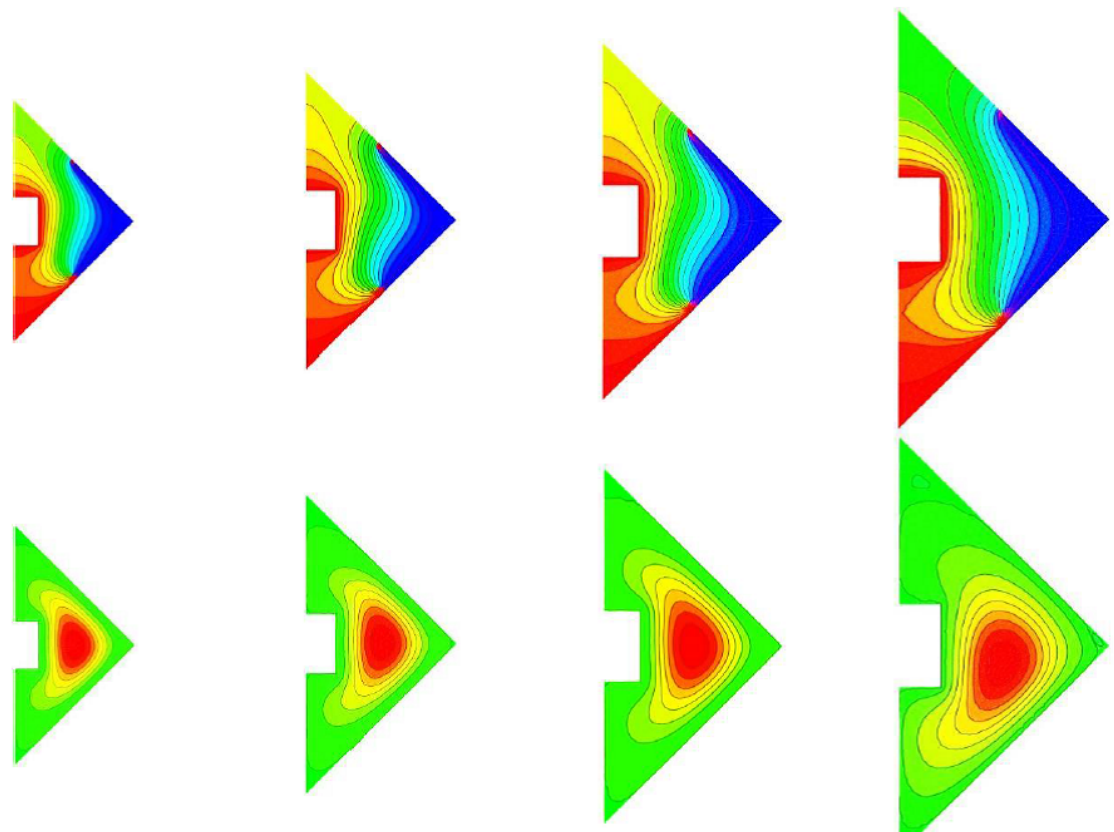

$x=10 \mathrm{~mm}$

$x=340 \mathrm{~mm}$

$x=680 \mathrm{~mm}$

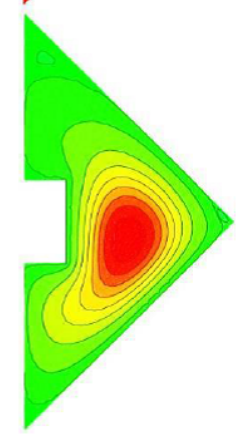

$\mathrm{Ra}=10^{6} \& \varepsilon=0.9 \& \phi=1 \%$
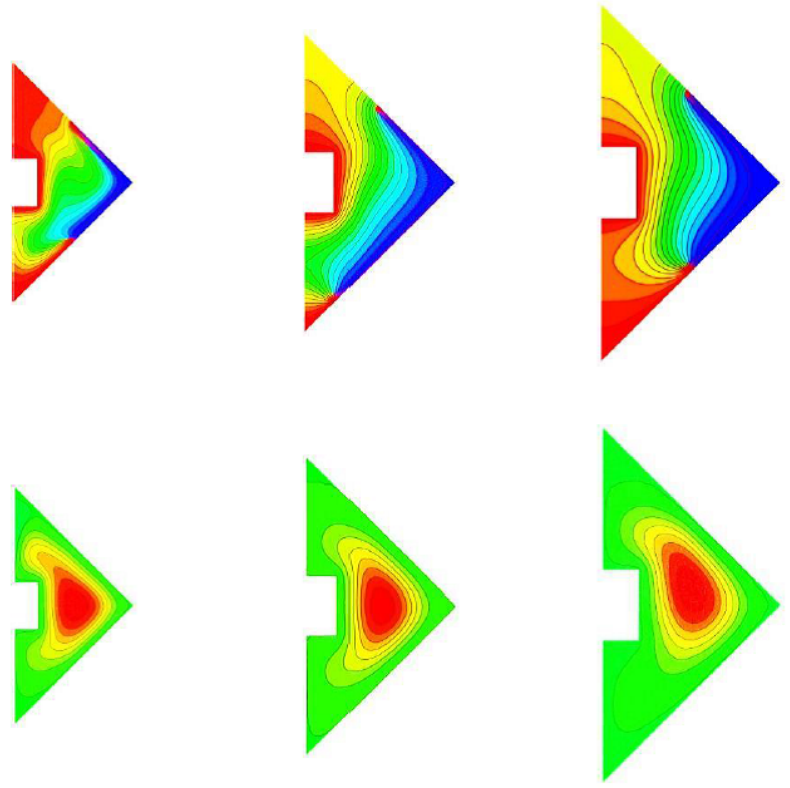

$x=10 \mathrm{~mm}$

$x=340 \mathrm{~mm}$

$x=680 \mathrm{~mm}$
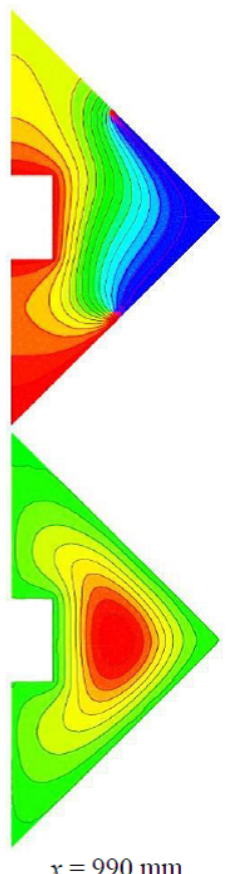

$\mathrm{Ra}=10^{6} \& \varepsilon=0.9 \& \phi=5 \%$ 

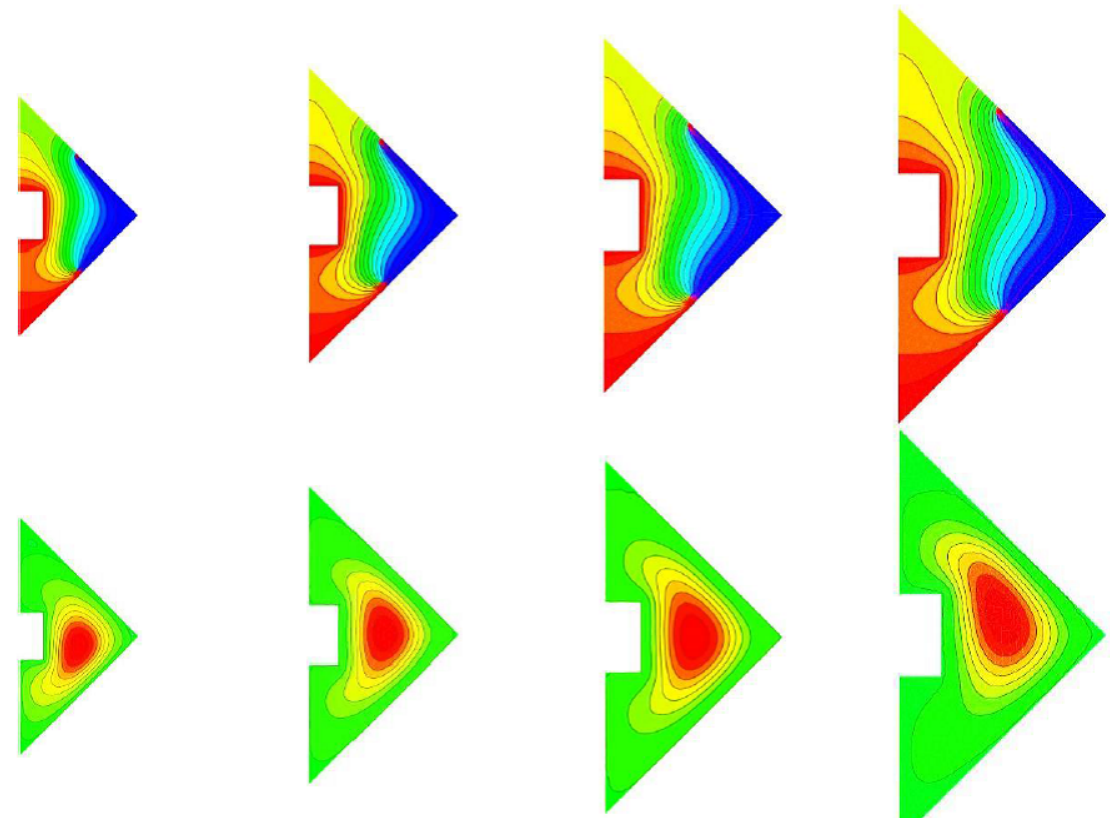

$$
x=10 \mathrm{~mm}
$$$$
x=340 \mathrm{~mm}
$$

$x=680 \mathrm{~mm}$

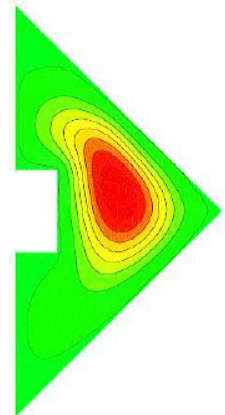

$\mathrm{Ra}=10^{8} \& \varepsilon=0.9 \& \phi=1 \%$
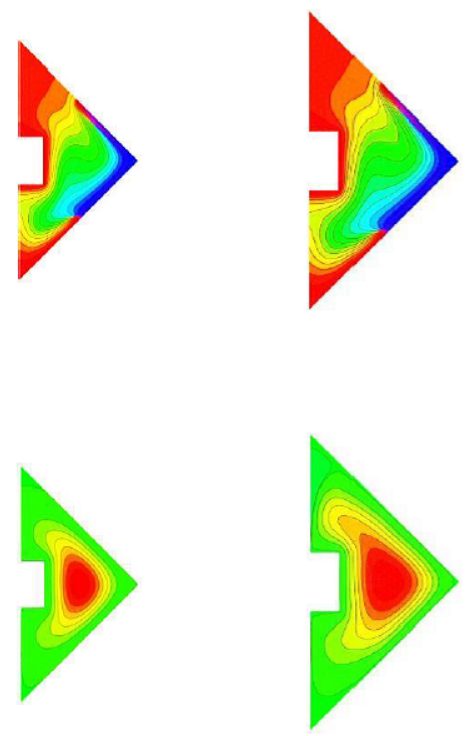

$$
x=10 \mathrm{~mm}
$$
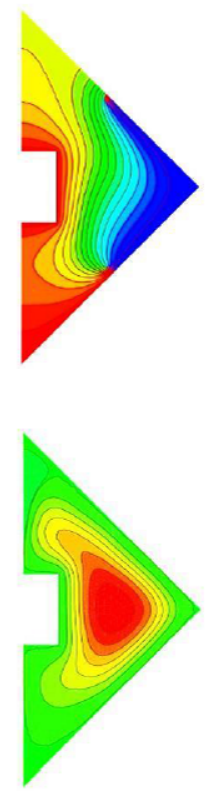

$x=680 \mathrm{~mm}$
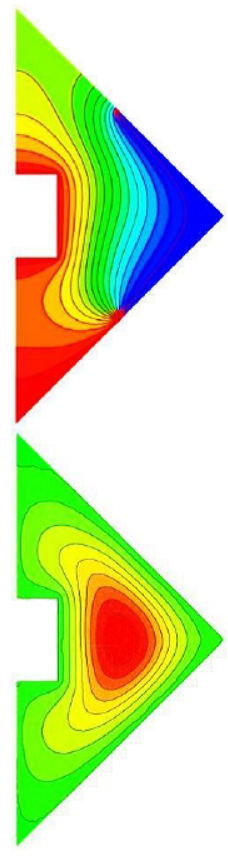

$x=990 \mathrm{~mm}$

$\mathrm{Ra}=10^{8} \& \varepsilon=0.9 \& \phi=5 \%$ 

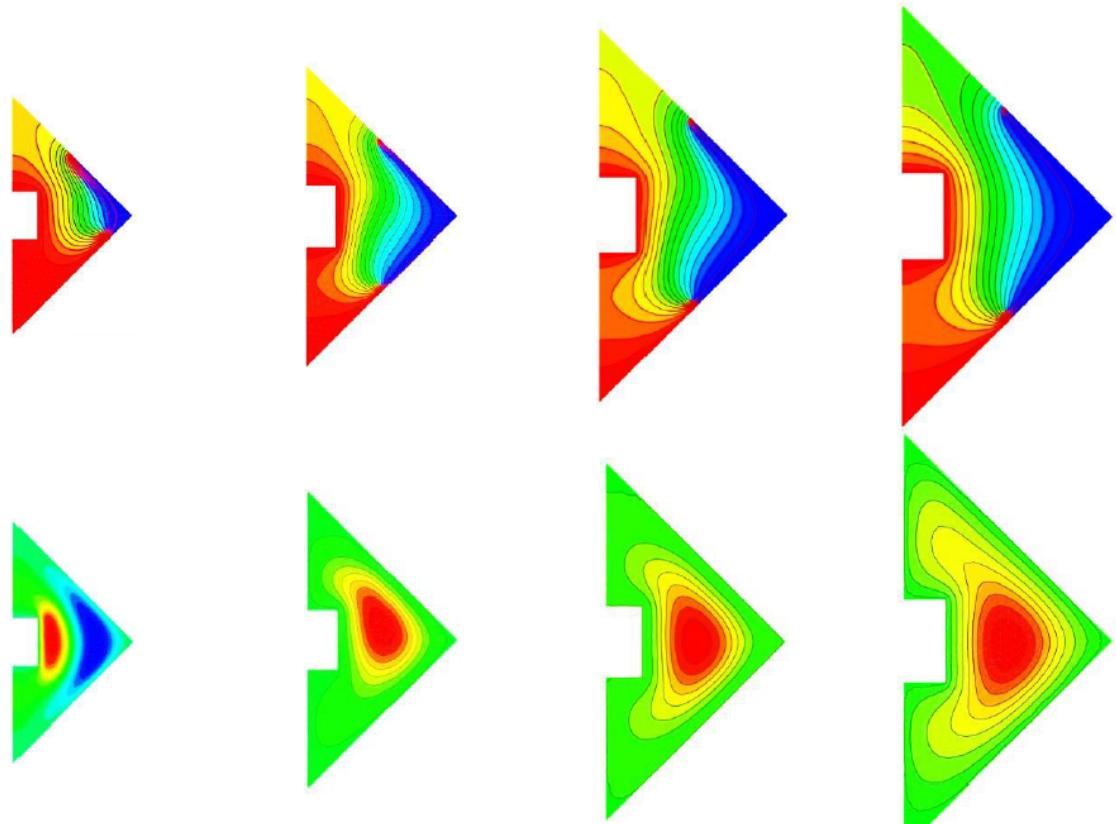

$x=10 \mathrm{~mm}$

$x=340 \mathrm{~mm}$

$x=680 \mathrm{~mm}$

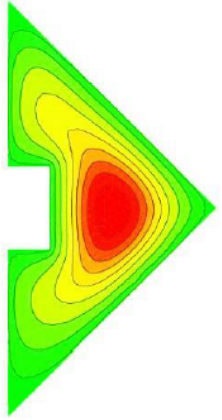

$x=990 \mathrm{~mm}$

$\mathrm{Ra}=10^{6} \& \varepsilon=0.9 \& \phi=1 \%$
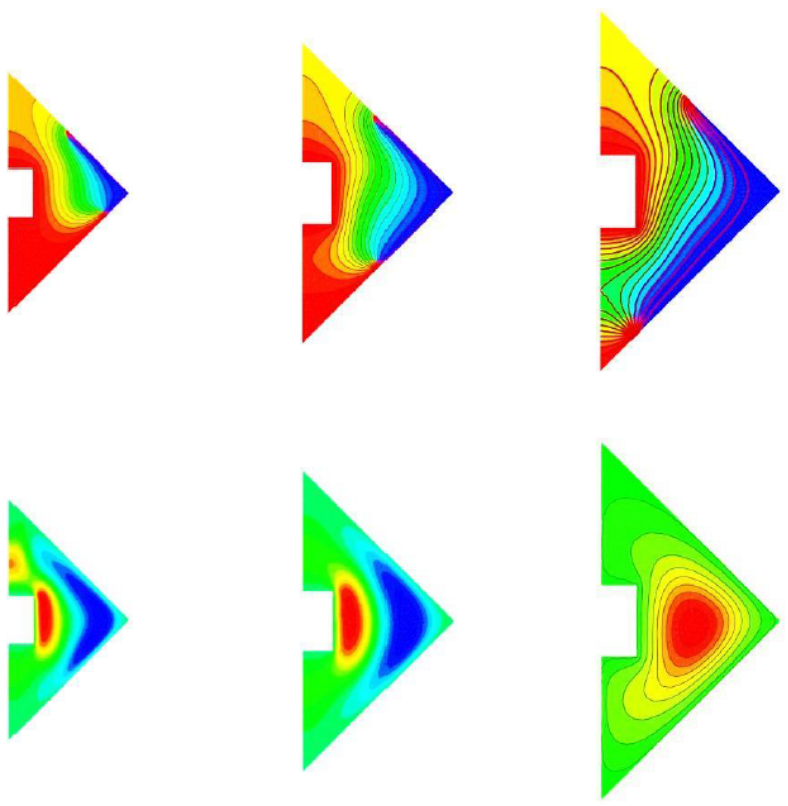

$x=10 \mathrm{~mm}$

$x=340 \mathrm{~mm}$

$x=680 \mathrm{~mm}$

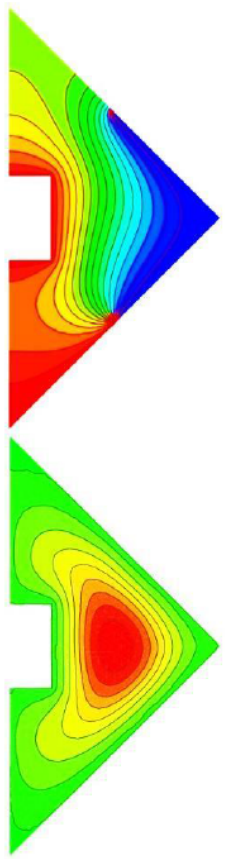

$\mathrm{Ra}=10^{6} \& \varepsilon=0.9 \& \phi=5 \%$ 

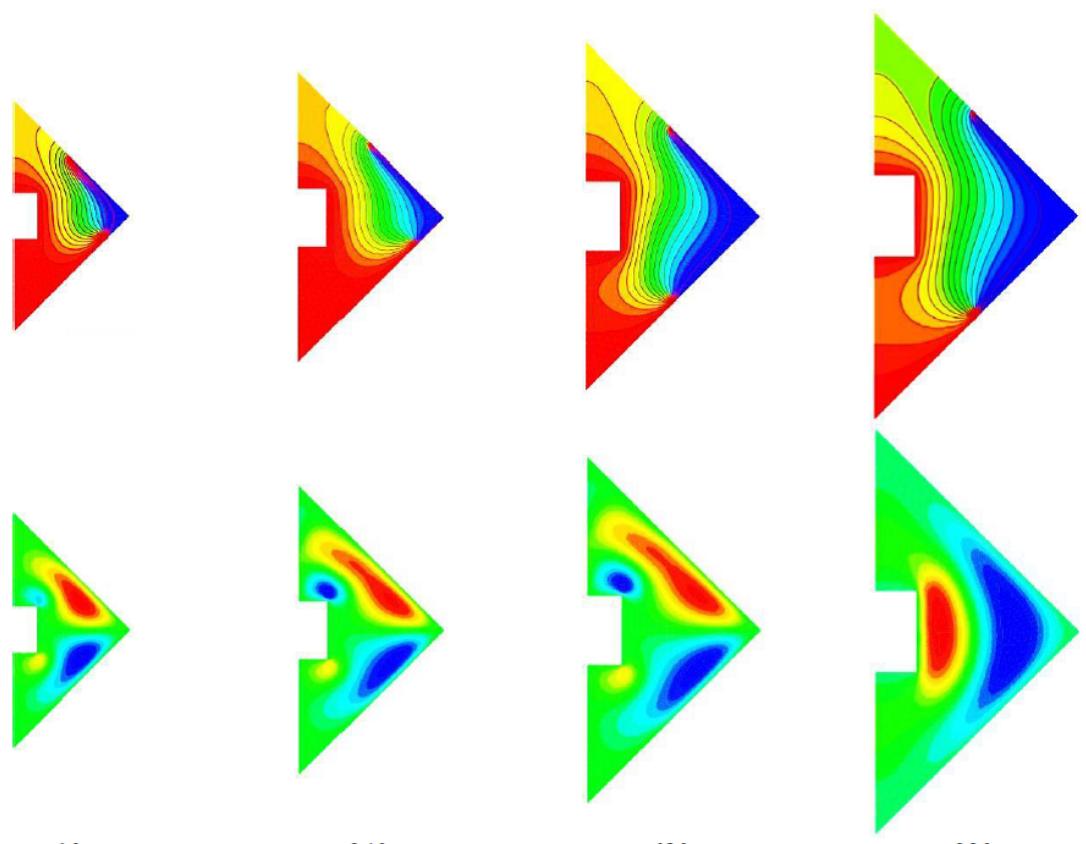

$x=10 \mathrm{~mm}$

$x=340 \mathrm{~mm}$

$x=680 \mathrm{~mm}$

$\mathrm{Ra}=10^{8} \& \varepsilon=0.9 \& \phi=1 \%$
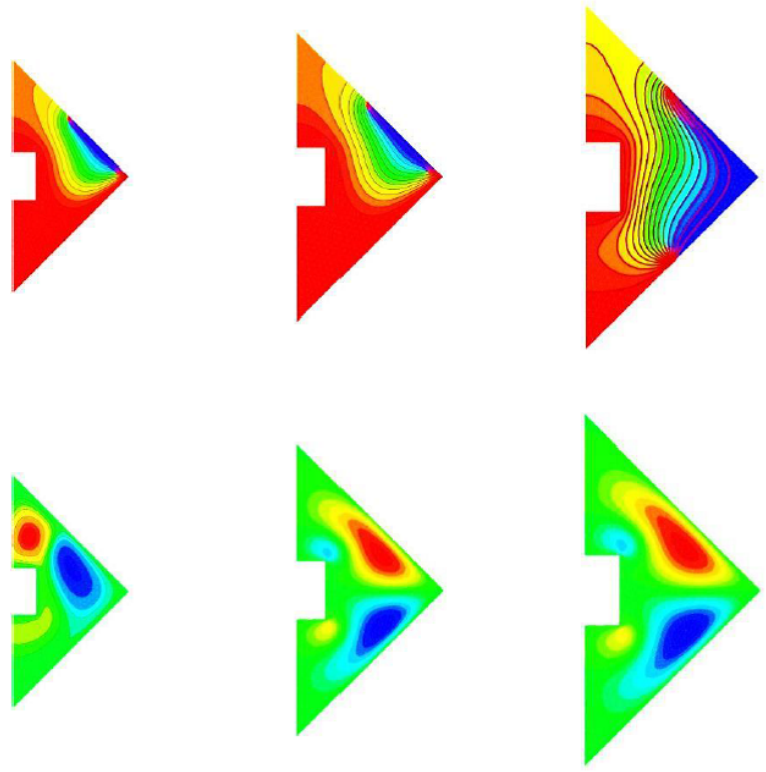

$x=10 \mathrm{~mm}$

$x=340 \mathrm{~mm}$

$x=680 \mathrm{~mm}$

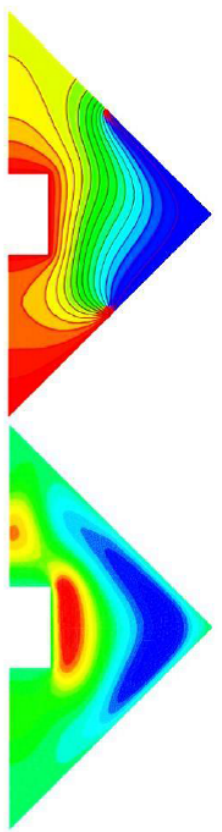

$\mathrm{Ra}=10^{8} \& \varepsilon=0.9 \& \phi=5 \%$ 


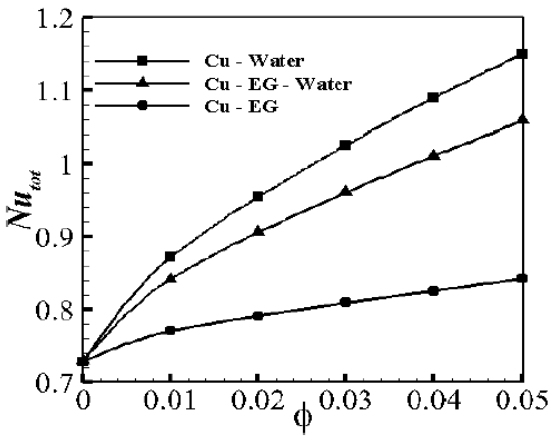

(a) $\mathrm{Ra}=10^{6}$ and $T_{h}=400$

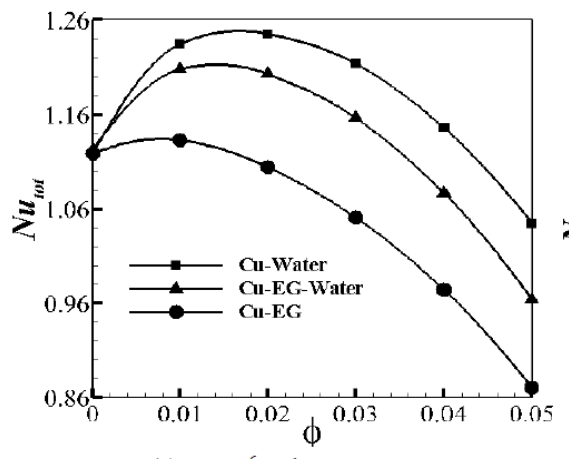

(c) $\mathrm{Ra}=10^{6}$ and $T_{h}=500$

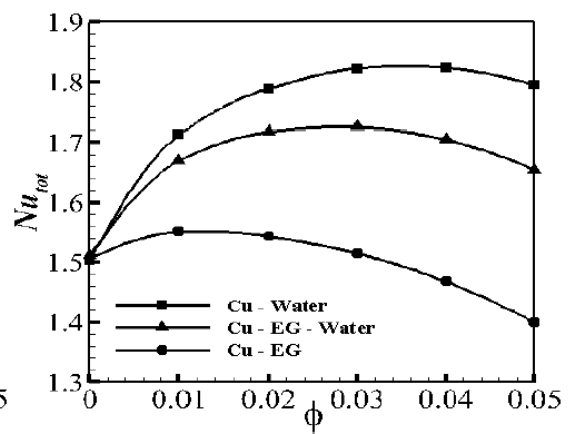

(b) $\mathrm{Ra}=10^{8}$ and $T_{h}=400$

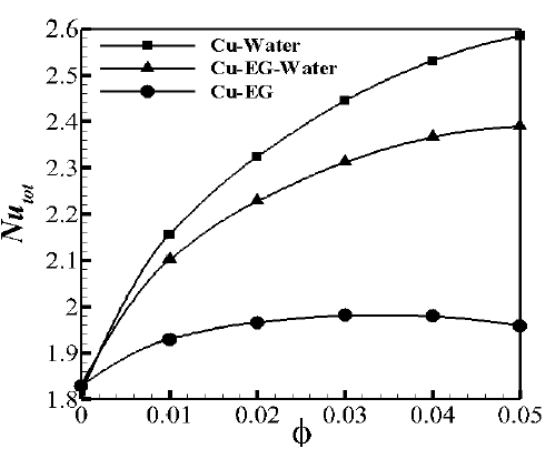

(d) $\mathrm{Ra}=10^{8}$ and $T_{h}=500$ 